\title{
Lesão por pressão: riscos para o desenvolvimento
}

\section{Pressure injury: development risks}

Lesión por presión: riesgos de desarrollo

ORCID: https://orcid.org/0000-0002-1560-5366 Centro Universitário Brasileiro, Brasil E-mail: isabeladasilva2036@gmail.com

Amanda Tayane Silva Galdino

ORCID: https://orcid.org/0000-0002-5480-2645 Centro Universitário Brasileiro, Brasil E-mail: amandatayanegg@gmail.com

Camilla Gomes da Silva Lacerda ORCID: https://orcid.org/0000-0003-1624-1821 Universidade Iguaçu, Brasil

E-mail: camillaglls@hotmail.com

Bernardo Lannes Ventura

ORCID: https://orcid.org/0000-0002-7012-4661 Universidade Iguaçu, Brasil

E-mail: bernardolannes25@gmail.com

Carla Gomes da Silva Lacerda

ORCID: https://orcid.org/0000-0002-9347-1308 Centro Universitário Redentor, Brasil E-mail: carla_gomes08@hotmail.com

Cicera Daniele Garcia Simões

ORCID: https://orcid.org/0000-0001-6992-9860 Centro Universitário de Juazeiro do Norte, Brasil E-mail: danielegsousa@hotmail.com

Laudilina Xavier Rocha Gonçalves ORCID: https://orcid.org/0000-0002-2352-7054 Centro Universitário de Juazeiro do Norte, Brasil E-mail: laudilinagonsalves@gmail.com

Carolina Francisca da Silva Ferreira ORCID: https://orcid.org/0000-0003-1955-0005 Centro Universitário de Juazeiro do Norte, Brasil E-mail: carollinadasilva@ hotmail.com

Janaina Cristina Sampaio Coelho Feitosa

ORCID: https://orcid.org/0000-0003-0832-9612 Hospital São Lucas, Brasil

E-mail: janacrisjcsc@hotmail.com

Emanuel Cardoso Monte ORCID: https://orcid.org/0000-0002-4719-7168 Centro Universitário de Juazeiro do Norte, Brasil E-mail: emanoelcm@gmail.com Crystianne Samara Barbosa Araújo ORCID: https://orcid.org/0000-0002-4168-7414 Centro Universitário de Juazeiro do Norte, Brasil E-mail: crystianne.barbosa@unijuazeiro.edu.br 


\author{
Cintia Nadhia Alencar Landim \\ ORCID: https://orcid.org/0000-0002-8189-9323 \\ Centro Universitário de Juazeiro do Norte, Brasil \\ E-mail: nadhia_landim@hotmail.com \\ Maria Elisa Regina Benjamin de Moura \\ ORCID: https://orcid.org/0000-0003-3145-4307 \\ Centro Universitário de Juazeiro do Norte, Brasil \\ E-mail: elisareg@gmail.com \\ Cicero Rafael Lopes da Silva \\ ORCID: https://orcid.org/0000-0001-8819-5380 \\ Centro Universitário de Juazeiro do Norte, Brasil \\ E-mail: rafael.lopes@unijuazeiro.edu.br
}

\begin{abstract}
Resumo
Objetivou-se compreender os riscos do desenvolvimento de lesões por pressão em idosos. Trata-se de uma revisão integrativa, realizada de abril a Julho de 2021. Utilizou-se os descritores: profissionais da área da saúde, lesão por pressão e saúde do idoso, nas bases: LILACS, MEDLINE e BVS. Encontraram-se 12 artigos, dos quais, foi evidenciado a importância de entender e qualificar a equipe de saúde para atuar de forma mais eficaz na intervenção da temática abordada, de forma a melhorar o atendimento, prevenindo o desenvolvimento dessas lesões. Através dos achados discutidos, se percebe de maneira esclarecida que as práticas da assistência tendem a reduzir o número de lesões por pressão, de forma a serem tratadas principalmente através da prevenção e do tratamento curativo.
\end{abstract}

Palavras-chave: Profissionais da saúde; Lesão por pressão; Saúde do idoso.

\begin{abstract}
The objective was to understand the risks of developing pressure injuries in the elderly. This is an integrative review, carried out from April to July 2021. The following descriptors were used: health professionals, pressure injuries and elderly health, in the bases: LILACS, MEDLINE and VHL. Twelve articles were found, of which the importance of understanding and qualifying the health team to act more effectively in the intervention of the topic addressed was highlighted, in order to improve care, preventing the development of these injuries. Through the findings discussed, it is clear that care practices tend to reduce the number of pressure injuries, in order to be treated mainly through prevention and curative treatment.
\end{abstract}

Keywords: Health professionals; Pressure injury; Health of the elderly.

\title{
Resumen
}

El objetivo era comprender los riesgos de desarrollar lesiones por presión en los ancianos. Se trata de una revisión integradora, realizada de abril a julio de 2021. Se utilizaron los siguientes descriptores: profesionales de la salud, lesiones por presión y salud del anciano, en las bases: LILACS, MEDLINE y VHL. Se encontraron doce artículos, de los cuales se destacó la importancia de comprender y capacitar al equipo de salud para actuar con mayor eficacia en la intervención del tema abordado, con el fin de mejorar la atención, previniendo el desarrollo de estas lesiones. A través de los hallazgos discutidos, es claro que las prácticas de cuidado tienden a reducir el número de lesiones por presión, para ser tratadas principalmente a través de la prevención y el tratamiento curativo.

Palabras clave: Profesionales de la salud; Lesión por presión; Salud de las personas mayores.

\section{Introdução}

A Lesão Por Pressão (LPP) é considerada um problema de saúde pública em todo o mundo devido à enorme carga que representa para os sistemas de saúde. Sua ocorrência é um evento ainda comum apesar da inserção de protocolos preventivos e da identificação precoce de pacientes com alto risco para o seu desenvolvimento (Ness et al., 2018).

Constitui-se como um agravo de importância epidemiológica, que acomete principalmente pacientes adultos, idosos, e/ou críticos, podendo causar importantes danos à saúde gerando sofrimento, contribuindo para o aumento do risco de complicações hospitalares, retardando a recuperação e prolongando o tempo de internação. Além disso, está relacionada a altos índices de mortalidade, aumento da carga de trabalho dos profissionais e dos custos para as instituições (Aldersen et al., 2017 \& Costa, Costa \& Boução, 2016).

Conforme estabelecido pelo National Pressure Ulcer Advisory Panel (NPUAP), a LPP caracteriza-se por ser um dano localizado na pele e/ou tecidos moles subjacentes, geralmente sobre uma proeminência óssea ou relacionado ao uso de dispositivo médico ou a outro artefato. Ela vem a ocorrer como resultado de uma pressão intensa e/ou prolongada em combinação com o 
cisalhamento (National Pressure Ulcer Advisory Panel, 2019).

O desenvolvimento da LPP é multifatorial, ou seja, vários fatores/causas, tendo como principais fatores predisponentes: déficit de atividade e/ou mobilidade, comprometimento sensorial ou cognitivo, deficiência nutricional, perfusão tissular inadequada, atrito e/ou fricção, umidade e o uso de dispositivos médicos que exercem uma pressão exacerbada e contínua sobre a pele (Edsberg et al., 2016).

A população idosa cresce juntamente com as comorbidades crônicas, trazendo um número relevante na população de idosos fragilizados, em condições que necessitam de auxílio e cuidados e de acordo com o nível de dependência são mantidos restritos ao leito. Dessa forma, evoluem para outras complicações, a exemplo da síndrome da imobilidade, que pode ser caracterizada como um complexo de sinais e sintomas resultantes da supressão de todos os movimentos articulares e, por conseguinte, da incapacidade da mudança postural, tendo como uma das principais consequências o surgimento de LPP (Almeida et al., 2019).

De acordo com o Ministério da Saúde (MS), grande parte das LPP podem ser evitadas com a adoção de medidas simples, como cuidados com a pele e mudança na posição do paciente no leito. Sendo assim, baseado no que diz os protocolos de prevenção de lesões por pressão, os profissionais de saúde devem implantar estratégias de prevenção, como garantir o reposicionamento do paciente e sua colocação em superfícies de redistribuição de pressão, para todos aqueles com risco identificado (Almeida, 2019).

O Protocolo de Prevenção de LPP provê recomendações a serem aplicadas a todos os indivíduos que estejam em risco de desenvolver lesões dessa natureza em distintos ambientes de cuidados. A presença da lesão é um indicador negativo de qualidade da assistência sendo prestada, sendo avaliada internacionalmente como um evento adverso, e representa importante desafio para o cuidado em saúde. Se faz recomendação que o profissional da área da saúde utilize ferramentas como a escala de Braden ou de Waterlow para identificação precoce das pessoas em risco de LPP, o que é fundamental para uma abordagem sistemática de prevenção e cuidados apropriados (Ministério da Saúde, 2013).

Pondera-se que a manutenção da integridade da pele de um idoso acamado tem por base o conhecimento e a aplicação de medidas preventivas relativamente simples, que devem ser praticadas pelo cuidador e pelos profissionais de saúde envolvidos na assistência do idoso vulnerável. As lesões por pressão podem ser evitadas por meio da identificação dos riscos e implantação de estratégias de prevenção para eliminar ou minimizar os fatores de riscos, como a avaliação diária da pele, manejo da umidade e redução da pressão local, em grande parte realizado pela equipe multiprofissional no ambiente hospitalar (Moro \& Caliri, 2016).

A ciência da prestação da assistência para e com esses pacientes, tem como objeto o cuidado. Nessa perspectiva podemos observar a importância do papel da equipe multiprofissional, que é fundamental para minimização ou até mesmo aniquilação das lesões por pressão. As lesões de pele ainda são um problema que desafia os profissionais de saúde (Soares \& Heidemann 2018).

As lesões de pele trazem muitos desafios para os profissionais de saúde na prática clínica, destacando-se a LPP, visto que, acometem principalmente os pacientes com restrição de mobilidade, ou seja, principalmente os acamados, isso acarreta o aumento de custos no tratamento e no tempo de hospitalização desses pacientes, proporcionando desconforto e desenvolvendo impacto negativo no serviço prestado e na qualidade de vida dos indivíduos (Freire et al., 2020).

Através do estudo da temática, surgiu-se a seguinte dúvida: Qual os aspectos que permeiam o desenvolvimento e a prevenção das Lesões Por Pressões em idosos?

Dessa forma, estabeleceu-se como objetivo deste estudo: Compreender os riscos do desenvolvimento de lesões por pressões em idosos. 


\section{Metodologia}

Trata-se de uma revisão integrativa da literatura, de cunho descritiva e com abordagem qualitativa, que segundo os autores Mendes, Silveira \& Galvão (2008), a mesma é desenvolvida através da realização das seguintes etapas: identificação do tema e seleção da questão norteadora da pesquisa; Investigação da produção científica que atenda a questão norteadora, conforme critérios de inclusão e exclusão; Coleta de dados; Análise dos dados e por fim, a elucidação dos dados encontrados e a apresentação da revisão.

Na perspectiva de obter dados apropriados ao propósito, realizou-se a busca nas bases de dados Literatura LatinoAmericana e do Caribe em Ciências da Saúde (LILACS) e na Medical Literature Analysis and Retrieval Sistem Online (MEDLINE) via Biblioteca Virtual em Saúde (BVS), com utilização dos Descritores em Ciências da Saúde (DECS): Profissionais da Saúde, Idoso e Lesão Por Pressão em associação ao operador booleano AND, durante os meses Abril à Julho de 2021.

Entre os critérios de elegibilidade, foram incluídos: artigos com disponibilidade gratuitamente na íntegra, com publicação nos idiomas português, inglês e espanhol, publicados no período de 2016 a 2021 que abordassem a temática do estudo e possibilitaram responder à questão norteadora desta pesquisa e seleção do título que contenha referências aos descritores usados na pesquisa.

Foram excluídos estudos com animais e/ou inconclusivos, estudos que não foram disponibilizados na íntegra, idiomas diferentes do critério de inclusão, título que não condizem com os descritores, além daqueles que apresentaram duplicidade na pesquisa e texto sem real relevância para o escopo do estudo.

Esta fase de seleção foi realizada pelos pesquisadores de forma independente, inicialmente por meio da adequação dos títulos e resumos à proposta do estudo onde após leitura, os dados foram inseridos em um instrumento elaborado pelos autores utilizando como base o protocolo prisma (Figura 1).

$\mathrm{Na}$ etapa de avaliação, foi realizada uma análise crítica dos estudos, a interpretação dos dados baseando-se na literatura vigente levando a discussão entre os autores 
Figura 1: Fluxograma de eleição dos estudos.

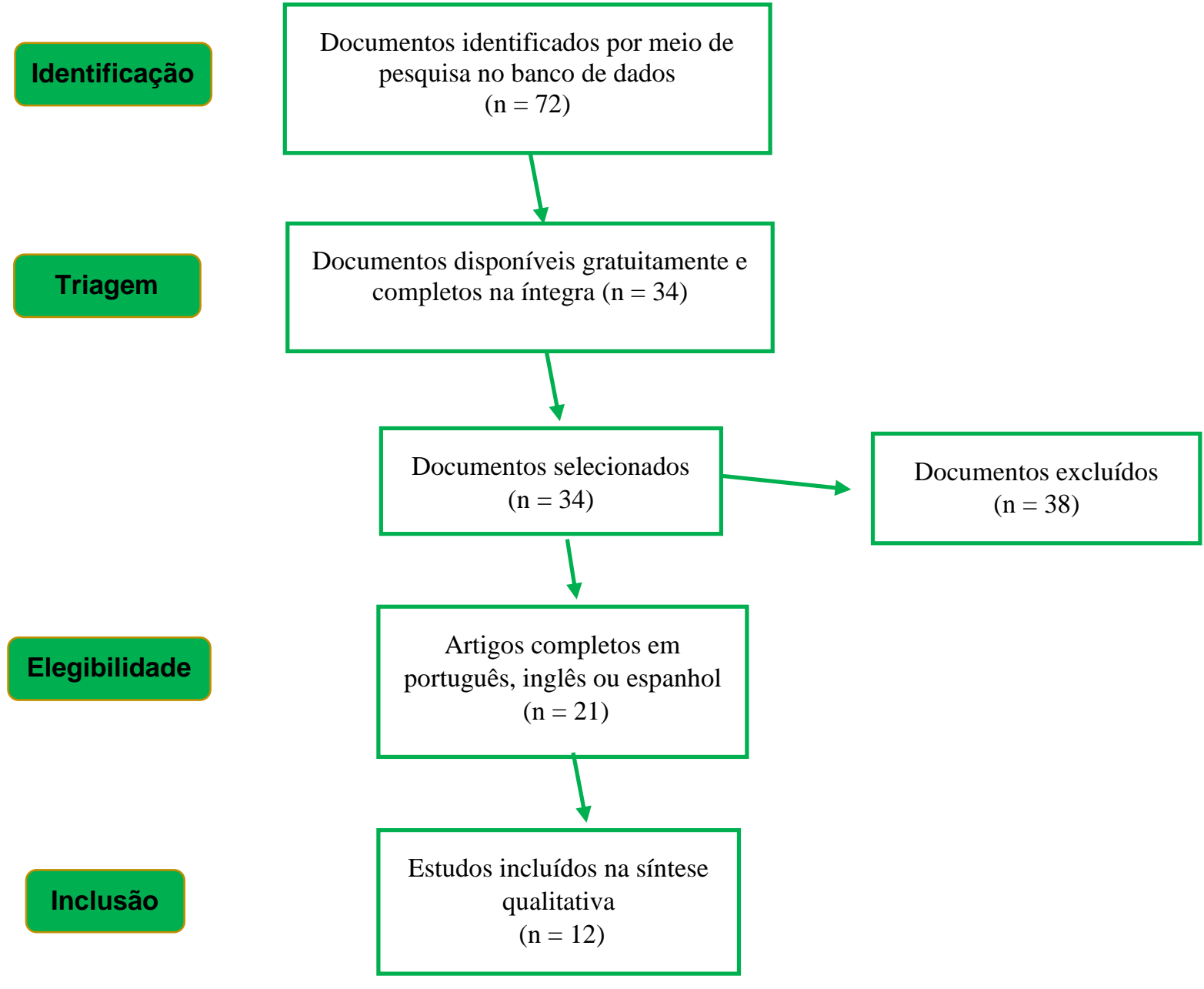

Fonte: Protocolo PRISMA.

A Figura 1 demonstra que apesar de terem sido encontradas 72 publicações para os descritores nos bancos de dados já mencionados, desses, 34 apresentavam textos na integra, dos quais 21 foram publicados em português, 07 em inglês e 06 em espanhol, dos quais apenas 12 adequaram-se ao período de publicação e apresentaram títulos condizentes com os descritores, sendo assim incluídos na amostra.

\section{Resultados}

Dos estudos foram extraídas informações como: número do achado, autor, ano, objetivo e amostra onde esses traziam dados referentes a cada artigo usado durante a elaboração do estudo, sendo tais informes inseridos no Quadro 1: 
Quadro 1: Caracterização da amostra do estudo. Juazeiro do Norte, CE.

\begin{tabular}{|c|c|c|c|}
\hline $\mathbf{N}^{\circ}$ & AUTOR/ANO & OBJETIVO & AMOSTRA \\
\hline 01 & $\begin{array}{l}\text { Cauduro et al., } \\
2018 .\end{array}$ & $\begin{array}{l}\text { Conhecer a atuação dos enfermeiros no } \\
\text { cuidado aos pacientes com lesões de pele. }\end{array}$ & $\begin{array}{l}\text { Entrevistas com roteiro semiestruturado com } 21 \\
\text { enfermeiros, analisadas pela Técnica de Análise de } \\
\text { Conteúdo na modalidade Análise Temática. }\end{array}$ \\
\hline 02 & Souza et al., 2017 & $\begin{array}{l}\text { O estudo teve como objetivo identificar na } \\
\text { literatura científica fatores predisponentes para } \\
\text { o surgimento das Lesões por pressão (LP) em } \\
\text { pacientes idosos. }\end{array}$ & $\begin{array}{l}\text { Nos } 21 \text { artigos analisa-dos houve predominância de risco } \\
\text { para desenvolvimento das LP no sexo feminino, e em } 9 \\
\text { artigos a variável avanço da idade estava relacionada aos } \\
\text { fatores de risco. }\end{array}$ \\
\hline 03 & Freire et al., 2020 & $\begin{array}{l}\text { Identificar na literatura científica as variáveis } \\
\text { associadas à prevenção da LPP para subsidiar } \\
\text { o cuidado de enfermagem. }\end{array}$ & $\begin{array}{l}\text { Foram } 18 \text { artigos analisados, destes, } 13 \text { eram } \\
\text { exclusivamente elaborados por enfermeiros. }\end{array}$ \\
\hline 04 & $\begin{array}{l}\text { Almeida et al., } \\
2019\end{array}$ & $\begin{array}{l}\text { Analisar a produção científica sobre a } \\
\text { assistência de enfermagem com vista à } \\
\text { prevenção de lesões por pressão em pacientes } \\
\text { hospitalizados. }\end{array}$ & 11 artigos foram incluídos na pesquisa e analisados. \\
\hline 05 & $\begin{array}{l}\text { Cardoso et al., } \\
2019 .\end{array}$ & $\begin{array}{l}\text { Avaliar o conhecimento dos enfermeiros sobre } \\
\text { Lesão por Pressão. }\end{array}$ & $\begin{array}{l}\text { Realizado com } 26 \text { Enfermeiros que compõem o quadro } \\
\text { funcional de todos os setores de um Hospital Privado em } \\
\text { Teresina-PI. }\end{array}$ \\
\hline 06 & $\begin{array}{l}\text { Soares e } \\
\text { Heidemann et al., } \\
2018 .\end{array}$ & $\begin{array}{l}\text { Apresentar a aplicabilidade da Escala de } \\
\text { Braden na percepção dos enfermeiros da } \\
\text { atenção primária, e identificar as medidas de } \\
\text { prevenção, e promoção da saúde. }\end{array}$ & $\begin{array}{l}\text { Pesquisa convergente assistencial, realizada num distrito } \\
\text { sanitário do Sul do Brasil, com } 20 \text { enfermeiros. A coleta de } \\
\text { dados ocorreu em julho de } 2014 \text {. }\end{array}$ \\
\hline 07 & $\begin{array}{l}\text { Ferraz \& Silva, } \\
2021\end{array}$ & $\begin{array}{l}\text { Avaliar a compreensão da equipe de } \\
\text { enfermagem a respeito da segurança do } \\
\text { paciente idoso hospitalizado. }\end{array}$ & $\begin{array}{l}\text { Estudo transversal, descritivo e de enfoque quantitativo } \\
\text { desenvolvido em uma unidade de clínica médica de um } \\
\text { hospital público do Distrito Federal. }\end{array}$ \\
\hline 08 & Silva et al., 2021 & $\begin{array}{l}\text { Avaliar a incidência de lesão por pressão (LPP) } \\
\text { em pacientes internados na emergência e } \\
\text { identificar os fatores de risco associados. }\end{array}$ & $\begin{array}{l}\text { Estudo prospectivo, longitudinal, observacional. Os dados } \\
\text { foram coletados por } 60 \text { dias consecutivos, utilizando-se } \\
\text { formulário próprio, inspeção da pele e prontuário. Amostra } \\
\text { constituída de } 225 \text { pacientes }\end{array}$ \\
\hline 09 & Lopes et al., 2020 & $\begin{array}{l}\text { Identificar os tipos de medicamentos mais } \\
\text { utilizados por idosos hospitalizados e } \\
\text { relacioná-los com o risco de desenvolvimento } \\
\text { de lesão por pressão. }\end{array}$ & $\begin{array}{l}\text { Amostra constituiu-se por } 48 \text { prontuários e a coleta de } \\
\text { dados ocorreu no período de agosto de } 2015 \text { a fevereiro de } \\
2016 \text {. }\end{array}$ \\
\hline 10 & Vieira et al., 2018 & $\begin{array}{l}\text { Avaliar o risco de idosos institucionalizados } \\
\text { com comprometimento na realização das } \\
\text { atividades de vida diárias (AVDs) } \\
\text { desenvolverem lesão por pressão (LPP). }\end{array}$ & $\begin{array}{l}\text { Amostra de } 44 \text { idosos, que utilizou como instrumentos de } \\
\text { coleta de dados questionário sobre perfil sociodemográfico } \\
\text { dos idosos, escala de Katz e escala de Braden. }\end{array}$ \\
\hline 11 & $\begin{array}{l}\text { Laranjeira \& } \& \\
\text { Loureiro, } 2017\end{array}$ & $\begin{array}{l}\text { Identificar e caracterizar os fatores de risco das } \\
\text { úlceras de pressão (UP) em doentes portadores } \\
\text { de UP, internados num Hospital Central } \\
\text { Português. }\end{array}$ & $\begin{array}{l}\text { Estudo descritivo de corte transversal com uma amostra } \\
\text { constituída de } 34 \text { indivíduos, } 70,6 \% \text { do sexo masculino e } \\
29,4 \% \text { do sexo feminino com média de idade de } \\
78,93 \pm 6,05 \text { anos. }\end{array}$ \\
\hline 12 & $\begin{array}{l}\text { Toffoletto et al., } \\
2016\end{array}$ & $\begin{array}{l}\text { Identificar os fatores relacionados à ocorrência } \\
\text { de eventos adversos em pacientes idosos } \\
\text { críticos internados em Unidade de Terapia } \\
\text { Intensiva segundo características } \\
\text { demográficas e clínicas. }\end{array}$ & $\begin{array}{l}\text { Estudo de coorte retrospectivo realizado em nove unidades } \\
\text { de um hospital universitário. Os dados foram coletados dos } \\
\text { prontuários e do acompanhamento de passagens de plantão } \\
\text { de enfermagem. }\end{array}$ \\
\hline
\end{tabular}

Fonte: Autores.

A partir desses artigos utilizados, pode se notar a importância do conhecimento e da assistência dos profissionais da área da saúde frente ao paciente idoso portador de LPP. O Quadro 02 expõe os principais achados de cada artigo, sendo utilizada 
a associação correspondente do número do artigo no Quadro 1 ao número do achado no Quadro 2. Alguns principais achados são descritos a seguir:

Quadro 2: Principais achados dos estudos incluídos na amostra.

\begin{tabular}{|c|c|}
\hline $\mathrm{N}^{\circ}$ DO ACHADO & PRINCIPAIS ACHADOS \\
\hline 01 & $\begin{array}{l}\text { Considerou-se o aprimoramento do enfermeiro, pela educação permanente, pelo trabalho em equipe e pelo auxílio } \\
\text { da Comissão de Prevenção e Tratamento de Feridas, uma estratégia para o cuidado da pele, analisando as condutas } \\
\text { dos enfermeiros e direcionando a prática profissional para o cuidado coletivo. }\end{array}$ \\
\hline 02 & $\begin{array}{l}\text { Ressalta-se, assim, que as lesões por pressão constituem um sério problema comumente identificável em idosos } \\
\text { tanto hospitalizados quanto em cuidado domiciliar e em instituições de longa permanência, especialmente quando } \\
\text { eles apresentam comprometimento de sua capacidade funcional, evidenciando, a partir das observações, a } \\
\text { relevância para a prática clínica e para o cuidado de enfermagem com ênfase na assistência integral e } \\
\text { individualizada. }\end{array}$ \\
\hline 03 & $\begin{array}{l}\text { Permitiu verificar que os cuidados voltados a medidas de prevenção se constituem como um tema que demanda } \\
\text { preocupação por parte da equipe multiprofissional, principalmente, pelos enfermeiros que têm investido de forma } \\
\text { expressiva no melhor entendimento do problema e na busca de soluções através de pesquisas metodologicamente } \\
\text { mais refinadas. }\end{array}$ \\
\hline 04 & $\begin{array}{l}\text { Evidenciaram a importância de padronizar as notificações e qualificar a equipe de enfermagem para atuar de forma } \\
\text { mais eficaz na intervenção da temática abordada. }\end{array}$ \\
\hline 05 & $\begin{array}{l}\text { Os principais resultados apontam para uma conformidade entre as ações e as recomendações da literatura, no } \\
\text { entanto acredita-se que é necessário investir em educação permanente sobre atualidades em coberturas/curativos, } \\
\text { para que os profissionais de enfermagem tenham um embasamento científico mais significativo que permita atuar } \\
\text { com segurança. }\end{array}$ \\
\hline 06 & $\begin{array}{l}\text { Uma avaliação adequada, um plano de cuidados que possa prevenir a lesão por pressão, assim como práticas que } \\
\text { promovam saúde, configuram-se como possibilidades criativas versus desafios, na inclusão de um novo paradigma } \\
\text { na atenção primária. }\end{array}$ \\
\hline 07 & $\begin{array}{l}\text { A comunicação, sistematização de fluxos, sobrecarga no trabalho, a cultura da segurança do paciente como medida } \\
\text { educativa e não punitiva são elementos que podem ser trabalhados na gestão da equipe de enfermagem para o } \\
\text { cuidado ao idoso hospitalizado. }\end{array}$ \\
\hline 08 & $\begin{array}{l}\text { Ressalta-se a necessidade de implementação de protocolos de prevenção de LPP nos serviços de emergência, além } \\
\text { da promoção de educação permanente para que a equipe de saúde direcione suas práticas para identificação de } \\
\text { riscos e segurança do cuidado. }\end{array}$ \\
\hline 09 & $\begin{array}{l}\text { Os tipos de medicamentos mais utilizados nos idosos deste estudo estavam diretamente relacionados às alterações } \\
\text { no sistema circulatório do idoso, o que compromete o risco para desenvolvimento de Lesão por Pressão. }\end{array}$ \\
\hline 10 & $\begin{array}{l}\text { A utilização de escalas preditivas como as de Braden e de Katz proporciona parâmetros para o enfermeiro planejar } \\
\text { cuidados com a pele de modo individualizado, visando a segurança e bem-estar dos idosos institucionalizados. }\end{array}$ \\
\hline 11 & $\begin{array}{l}\text { Verificou-se que os indivíduos com LPP já apresentavam LPP à entrada no serviço onde estavam hospitalizados; } \\
\text { os indivíduos, na sua maioria, têm um tempo de internamento de } 6 \text { dias a } 1 \text { mês e quanto maior é o tempo de } \\
\text { internamento mais elevado é o número de LPP que se desenvolvem nos indivíduos. }\end{array}$ \\
\hline 12 & $\begin{array}{l}\text { A identificação dos eventos e fatores associados no idoso subsidiam a prevenção dessas ocorrências perante as } \\
\text { vulnerabilidades dessa faixa etária. }\end{array}$ \\
\hline
\end{tabular}

Fonte: Autores.

\section{Discussão}

A LPP é motivo de grande preocupação por parte da equipe da área de saúde, onde os profissionais da área da saúde são os maiores envolvidos no tratamento direto a este agravo, por passarem maior tempo com os pacientes hospitalizados. 
Antigamente era chamada de úlcera por pressão, porém houve alteração na terminologia úlcera por pressão (UPP), para lesão por pressão (LPP), pois no estágio 1 e na tissular profunda ainda não há desenvolvimento de uma úlcera, segundo Edsberg et al (2016): “O consenso foi usado para esclarecer ainda mais os aspectos de cada uma das definições" (Lamão, Quintão \& Nunes, 2016).

Essa classificação é extremamente relevante para evolução do quadro de saúde do paciente, porém a pesquisa de Cardoso et al (2019) descreve que menos de 10\% dos profissionais participantes acertaram acima de $90 \%$ das questões sobre as LPP's. A incidência também está relacionada ao conhecimento da equipe para a sua prevenção, então é uma peça fundamental durante a prestação do cuidado, então se o profissional da saúde apresenta déficit nesse assunto, pode levar ao desenvolvimento pela ausência de prevenção das causas ou uma de mudança/piora na classificação da mesma (Campoi et al., 2019).

O profissional da saúde é parte de uma equipe multidisciplinar, sendo líder e gestor do cuidado, responsável pelas tomadas de decisões proporcionando a melhor prática do cuidado a ser empregada nos pacientes hospitalizados, sempre buscando qualidade na assistência prestada, então acaba sendo o profissional da área da saúde de destaque para a prestação de cuidados nesses casos e consequentemente carece de uma boa educação de base sobre a temática abordada, então este deve buscar estudos, realizar educações em saúde com enfoque de ensinar ao paciente a prevenir e a manter os cuidados, mantendo o raciocínio crítico e estimulando o cuidado por toda a equipe multiprofissional (Cauduro et al., 2018 \& Souza \& Faustino, 2019).

A combinação das escalas de avaliação de risco com o raciocínio clínico auxilia os profissionais a construir intervenções mais adequadas para o paciente. A avaliação deve ser realizada assim que possível, preferencialmente na admissão do paciente, e repetida quando houver mudanças nas condições de saúde do paciente. A escala de Braden é a mais utilizada no mundo, sendo traduzida e validada no Brasil, e tendo sua utilização divulgada em contextos de prática e pesquisas (Vasconcelos \& Caliri, 2017).

Uma das causas para o desenvolvimento é a idade, por exemplo, os idosos são mais suscetíveis ao desenvolvimento das LPP's, devido às condições causadas pelo envelhecimento do ser humano, como a rigidez do colágeno, diminuição da espessura da pele e das fibras elásticas. Incluindo ainda, a diminuição dos capilares da derme, redução do tecido adiposo subcutâneo nos membros, podendo ocasionar a desidratação da pele e diminuição dos suplementos sanguíneos, ou seja, alguns dos fatores predisponentes ao aparecimento de lesões (Souza, Freire \& Souza 2017).

Como a idade é um dos fatores desencadeantes, cabe a equipe de saúde a realização de medidas de segurança do paciente, como a criação de um plano de cuidados voltados para a prevenção e específicos para esse tipo de lesão. No estudo de Ferraz \& Silva (2021) verifica-se que mais de 16\% dos participantes da pesquisa, não montavam um plano para essas assistências.

Dentre os principais fatores determinantes para o aparecimento dessas lesões, é possível citar: Indivíduos institucionalizados ou que não possuem presença de um membro cuidador, Incontinências, Doenças crônicas (Hipertensão Arterial Sistêmica e Diabetes Mellitus), uso de medicações/psicofármacos e longos períodos de internação (Laranjeira \& Loureiro, 2017). Adentrando esses psicofármacos, baseado na pesquisa desenvolvida por Lopes et al (2020), os de maiores prevalências em relação ao desenvolvimento de LPP são: anti-hipertensivos, analgésicos, diuréticos, drogas vasoativas e antibióticos.

As LPP's apresentam-se como um problema de saúde de bastante significado, podendo refletir negativamente e causar eventos adversos, nesse caso ocorre o evento de lesão não intencional, afetando a qualidade de vida da pessoa acometidas, além de influenciar durante a internação desse paciente, pois pode aumentar o tempo que ele irá passar internado, trazer maiores sensações de desconforto e dores constantes, impactando em todo o processo de cuidar (Santos et al, 2019 \& Teixeira et al., 2018 \& Toffoletto et al., 2016). 


\section{Considerações Finais}

Diante dos dados apresentados, nota-se a importância da atenção dos profissionais de saúde com realização das medidas de segurança para o paciente de forma a prevenir o aparecimento de LPP's, especialmente nos idosos acamados que vão apresentar vários fatores para o desenvolvimento dessa lesão. Uma atenção maior para fatores predisponentes passa pela boa avaliação e concentração do profissional durante o cuidado ao paciente seja hospitalizado ou em domicílio.

Através dos achados discutidos, se percebe de maneira esclarecida que as práticas da assistência durante a prestação do cuidado e em conjunto com o raciocínio clínico e tabelas para avaliação de risco tendem a reduzir o número de lesões por pressão, de forma a serem tratadas principalmente através da prevenção e do tratamento curativo. Porém, em contraste a isso o alto número de prevalências de casos ainda encontrados, faz com que esse problema esteja longe de ser solucionado e continue sendo algo significativamente negativo na saúde pública (Soares \& Heidemann 2018).

Enfatiza-se, por fim, a importância da assistência qualificada pelo profissional da saúde, de maneira que venha a oferecer o melhor cuidado possível se atentando a todos os cuidados necessários e tendo a atenção necessária para enxergar os riscos dos pacientes, aplicando o conhecimento adquirido de forma a sempre focar no cuidado do paciente.

\section{Referências}

Alderden, J., et al. (2017). Risk factors for pressure injuries among critical care patients: A systematic review. Int J Nurs Stud., 71: 97-114.

Almeida, F., et al. (2019). Assistência de Enfermagem na prevenção da lesão por pressão: uma revisão integrativa. Revista Eletrônica Acervo Saúde/Eletronic Journal Collection Health. 30: 1-9.

Campoi, A. L. M., et al. (2019). Educação permanente para boas práticas na prevenção de lesão por pressão: quase-experimento. Rev. Bras. Enferm. 72 (6)

Cardoso, D. S., et al. (2019). Conhecimento dos Enfermeiros sobre Classificação e Prevenção de Lesão por Pressão. Rev. Fund. Care. Online. 11 (3): 560-566.

Cauduro, F. P., et al. (2018). Atuação dos Enfermeiros no Cuidado das Lesões de Pele. Rev. Enferm. UFPE on line., 12 (10): $2628-34$.

Costa, C. R., Costa, M. L., \& Boução, D. M. N. (2016). Braden scale: the importance of evaluation of pressure ulcer risk in patients in an intensive care unit. Revista Recien. 6 (17): 36-44.

Edsberg, L. E., et al. (2016). Revised National pressure ulcer advisory panel pressure injury staging system: revised pressure injury staging system. $J$ Wound Ostomy Continence Nurs. 43 (6): 585-597.

Ferraz, C. R. \& Silva, H. S. (2021). A Compreensão da Equipe de Enfermagem frente a segurança do Paciente Idoso Hospitalizado. Comunicação em Ciências da Saúde, 32 (1).

Freire, D. A., et al. (2020). Variáveis associadas à prevenção das lesões por pressão: Conhecimento para o cuidado de enfermagem. $R$. pesq.: cuid. fundam. Online, 12: 1172-1178.

Lamão, L. C. L., Quintão, V. A. \& Nunes, C. R. (2016). Cuidados de enfermagem na prevenção de lesão por pressão. Revista Científica Interdisciplinar. 1 (1).

Laranjeira, C. A. \& Loureiro, S. (2017). Fatores de risco das úlceras de pressão em doentes internados num hospital português. Rev. Salud pública 19 (1): 99 104.

Lopes, T. F., et al. (2020). Medicamentos e sua relação com o desenvolvimento de lesão por pressão em idosos hospitalizados. Rev Online Pesq Cuid Fundam. 12: $212-16$.

Ministério da Saúde. Anvisa. Fiocruz. (2013). Protocolo para prevenção de úlcera por pressão. PROQUALIS.

Mendes, K. D. S., Silveira, R. C. C. P. \& Galvão, C. M. Revisão integrativa: método de pesquisa para a incorporação de evidências na saúde e na enfermagem. Reflexão, Texto \& Contexto - Enfermagem, 17 (4).

Moro, J. V. \& Caliri, M. H. L. (2016). Úlcera por pressão após a alta hospitalar e o cuidado em domicílio. Esc Anna Nery. 20(3): 1-6.

National Pressure Ulcer Advisory Panel. (2019). Prevention and treatment of pressure ulcers/injuries: Quick reference guide. Emily Haesler (Ed.) EPUAP/NPIAP/PPPIA.

Ness, S. J., et al. (2018). The pressures of obesity: The relationship between obesity, malnutrition and pressure injuries in hospital inpatients. Clin Nutr., 37 (5): $1569-1574$.

Santos, T. A. V., et al. (2019). Assistencia de enfermagem ao paciente com lesão por pressão. Brazilian Journal of Surgery and Clinical Research - BJSCR, 27 (3): $120-124$. 
Research, Society and Development, v. 10, n. 13, e474101321389, 2021

(CC BY 4.0) | ISSN 2525-3409 | DOI: http://dx.doi.org/10.33448/rsd-v10i13.21389

Soares, C. F. \& Heidemann, I. T. S. B. (2018). Promoção da saúde e prevenção da lesão por pressão: Expectativas do enfermeiro da atenção primária. Texto Contexto Enferm, 27 (2): e1630016.

Souza, E., Freire, D. E. \& Souza, M. A. O. (2017). Fatores predisponentes para o desenvolvimento da lesão por pressão em pacientes idosos: uma revisão integrativa. Estima, 15 (4): $229-239$.

Teixeira, C. C., et al. (2018). Fatores relacionados à ocorrência de eventos adversos em pacientes idosos internados. Rev baiana enferm. 32 : e25772.

Toffoletto, M. C., et al. (2016). Fatores relacionados à ocorrência de eventos adversos em pacientes idosos críticos. Revista Brasileira de Enfermagem [online]. 69 (6): 1039-1045.

Vieira, V. A. S., et al. (2018). Risco de lesão por pressão em idosos com comprometimento na realização de atividades diárias. Revista de Enfermagem do Centro-Oeste Mineiro. 8: e2599. 